\title{
Ethical Considerations in Clinical Trials
}

\author{
Povl Riis \\ Copenhagen, Denmark
}

Ethical aspects of biomedical research in humans have been unmasked at a late stage in the history of science. They have been apparent for not more than approximately 60 years, and did not gain attention as a result of ongoing refinements of fundamental human rights. On the contrary, their recognition followed a traditional pattern in the history of human rights, in which the occurrence of severe, mass-scale atrocities is the tragic eye-opener, starting a global rebound reaction that leads to measures for regulation, control, education and prevention [1].

Now, after half a century's development, one can speak of not only a reaction, but a strong worldwide movement that has made ethics an obligatory component of scientists', editors', universities' and ministries' responsibility for the quality of research projects far beyond their technical qualities. However, the ethical demands in research have not only expanded over the world. An extension has also been seen in a temporal perspective for individual projects involving humans. Consequently, a research protocol today will not only include ethical considerations before the start of a project, but also during and after, and not only related to the research subjects but also to the researcher's reliability, i.e. his or her personal ethics.

Before initiating a project, the ethical control comprises assuring the project's accordance with international guidelines or conventions $[1,2]$ and with the home country's legislation, if the national development has led to such judicial regulation [3]. But irrespective of differences between national control structures, a given biomedical project involving humans will have to pass at least one so-called research ethical committee (sometimes internationally grouped as institutional review boards, an outdated general term, because now the most developed systems do not rely on institutional committees, but are built up of more independent committees with a much broader base than an institution).

A good starting point for clinical researchers is consequently to anticipate the analytic steps that most developed committee systems take today, even more and more generalized through, for instance, the Council of Europe's work via its Bioconvention [2] and the derived Protocol expected to be published in 2000 [4].

The first step is to evaluate the originality of a project's underlying idea. If it is very trivial, i.e. unlikely to generate any new knowledge, it might even fall at this stage, based on the saying that "every project involving man and not fulfilling the simplest scientific demands is in itself unethical, even if nonrisky'. Purely 'me too' trials represent such projects, with the only exception that an identical trial, number two or three after the original one, might be valuable in order to minimize the influence of statistical type one and type two errors.

Still another technical hurdle has to be overcome before the ethical analysis starts: the methodology chosen in order to procure a potential answer to the initial question. If the methods, the variables, the patient numbers, the bias control, the randomization and other key aspects are obviously invalid, the project will not proceed to the committee's next analytic step, even if the idea was original. Sometimes committees will try to help young scien-

\section{KARGER \\ Fax +4161306 1234 \\ E-Mail karger@karger.ch} www. karger.com (c) 2000 S. Karger AG, Basel

1422-9528/01/0011-0002\$17.50/0

Accessible online at:

www. karger.com/journals/hed
Prof. Povl Riis
Nerievej 7
DK-2900 Hellerup (Denmark) 
tists to improve the quality of their methodology, if possible, but their primary task is not to act as scientific advisers; consequently, this can only be done as a spin-off within the nonacceptance letter from the committee to the scientists. The number of projects handled in national systems, for instance, a national prevalence of 600 protocols per million inhabitants per year, or on average 350 protocols per committee per year, explains why this is so [5].

As an introduction to the ethical analysis, if the idea and the methodology are considered acceptable, the scientists must summarize the international literature, especially any results illustrating the ethical acceptability of using placebos in the control group, based on the "ethical axiom' that use of a placebo is only acceptable if no efficient treatments exist and are nationally accessible. Exemptions occur, but only in the case of less severe diseases and under very strict precautions determined by the committee.

The ethical analysis starts with a safety evaluation: do trial patients run an extra risk by participating? If therapeutic or diagnostic interventions are tested, will the intervention group and the control group have the same chance/risk to be winners/losers? If the project involves healthy volunteers, safety measures have to be documented with results approaching zero. Similarly strong precautions are demanded when trial patients are children, psychiatric patients or others who need extra protection [2].

The next step is an evaluation of the respect that will be shown potential participants, based on the two key terms information and consent. Information must be truthful and presented in an understandable form. Clinical scientists have been able to learn a way of writing that explains complicated clinical facts to lay people in simple, but nonetheless grown-up language, with the result that the last decades have shown a marked improvement in the patient-scientist relationship, which has even had a positive influence on the patient-doctor relationship outside clinical research.

The third aspect of the ethical analysis of research is the question of whether fundamental cultural or religious values within a country will be offended by the project. This is not meant as a kind of general censorship, but rather an inclusion of this aspect among the other pros and cons. Obviously, this last factor is most relevant, for instance, in projects on fertilized human eggs and pregnant women, but the possibility of socially stigmatizing groups of patients might also need to be considered, even in apparently ethically neutral projects, as, for instance, cardiovascular trials.

Ethical Considerations in Clinical Trials
Tracking a given trial project that has passed committee control still leads to other ethical considerations, i.e. those of publication ethics and the scientist's personal ethics. These have a large area of overlap, and can consequently be dealt with in common here. The scientist's credibility, i.e. his or her navigating at a safe distance from scientific dishonesty (in severe cases named scientific fraud), is an ongoing conditio sine qua non during the whole trial. The best means of prevention, especially in large clinical trials, is to invite only participants and centers that have an explicit reputation of high ethical standards in research, and to remember the bandwagon phenomenon, often evoked when large and prestigious multicenter trials are to be launched [6]. Another safety measure, not only applicable to good clinical practice monitoring in drug trials, is the practice of repetitive visits and data evaluation during the trial.

Reaching the promised land for a clinical researcher, the completion of the trial and the subsequent publication phase, does not mean that ethical problems are definitively left behind. On the contrary, publication ethics have proven to be an important part of the ethical spectrum, where interscientist and editor-scientist ethics, and not the patient-scientist ethics, are the important issue [6, 7]. If a trial has been successfully accomplished, severe discussions between all the scientists involved often create serious problems. Preventing this requires detailed decisions on these matters, written down in reports from the planning phase of the trial [7]. Aborting a trial because of quarrels between researchers in the publication phase not only constitutes a breach of scientists' ethics, but also of research ethics, because the altruistic participation of many trial patients will have thus been in vain.

In relation to the publishing journal, important ethical problems exist as a further part of publication ethics. Even if it is difficult for the scientist to positively influence them, he or she ought to know which ethical aspects are still relevant after the promising sound of the mailbox lid has been heard, as a sign of the trial's physical completion. The editor is, for instance, expected to be fast and fair in selecting manuscripts for publication. The referees must be similarly fast and fair, and must not lift ideas or combine such a theft with unrightful rejection of the original manuscript.

Based on the experiences of a national system controlling biomedical scientists' ethics, the most important aspect of this subarea of ethics is the prevalence of unrightful authorship, i.e. authors not fulfilling the authorship definition of the International Group of Medical Journal Editors [8]. In an attempt to transfer the observance of 
these demands, because they have not been respected, from the authors themselves to the editors, the Danish national control body has suggested the obligatory use of more stringent documentation for each author's contribution [7].

Finally, ethics related to trials based on joint venture arrangements between developed and underdeveloped countries will be briefly mentioned, based on a 20 years' tested handling in Denmark, and ongoing work in the US National Institute of Health and the British Nuffield Foundation. The basic ethical demand is that trials of major importance for populations of developed countries must not be transferred to developing countries for reasons of convenience or economy, but rather, trials hosted in these countries must primarily deal with diseases of major importance for the underdeveloped nation [3]. Fur- thermore, such joint venture projects must be approved officially in both the donor and recipient countries, and must include a number of local scientists and technicians, also as potential authors, consequently avoiding 'the flying scientist syndrome', i.e. the visiting scientist who only spends a very brief period in the developing country, enough to collect the necessary samples and leave by the earliest plane.

The long ethical journey briefly described above should not discourage clinical researchers, but instead show which aspects ought to be considered, especially in the planning phase of a study. Having duly considered and weighed these issues can only add to the deep and undisturbed satisfaction resulting from the accomplishment of original clinical research.

\section{References}

1 World Medical Association: The Declaration of Helsinki. Helsinki, WMA, 1975 (with subsequent additions)

2 The Council of Europe: Convention on Human Rights and Biomedicine. Oviedo, 1997.

3 Act No. 503, June 1992: Scientific Ethical Committee System and the Handling of Biomedical Research Projects. Copenhagen, The Danish Central Scientific Ethical Committee, Collection of Annexes, 1994.
4 The Council of Europe: Working Party on Biomedical Research (CDBI-CO-GT2): Draft Protocol on Biomedical Research (under preparation).

5 Central Scientific Ethical Committee of Denmark: Annual Report 1998. Copenhagen, Ministry of Research, 1999.
6 Riis P: Sociology and psychology within the scope of scientific dishonesty. Sci Eng Ethics 2000;6:35-39.

7 Committee on Scientific Dishonesty: Guideline for Agreements at the Initiation of Research Projects. Copenhagen, Ministry of Science, 1998

8 Committee on Scientific Dishonesty: Guidelines Concerning Authorship. Copenhagen, Ministry of Science, 1998. 